\title{
Review of: "Integration of deep learning and soft robotics for a biomimetic approach to nonlinear sensing"
}

\author{
lei wang $^{1}$ \\ 1 Shenzhen Institutes of Advanced Technology - Chinese Academy of Sciences \\ Potential competing interests: The author(s) declared that no potential competing interests exist.
}

An interesting paper 